\section{KOMPASS}

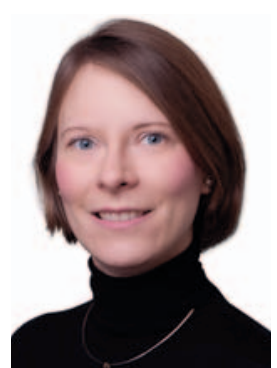

Nina Neuendorff,

Koordination «OnkoCampus»

\title{
Theorie und Praxis: Zusammen sind sie stark
}

Ich freue mich sehr, Ihnen nun in der vorliegenden Ausgabe zum zweiten Mal die Rubrik «OnkoCampus» präsentieren zu dürfen.

Fortgesetzt wird unsere Serie zur Vorstellung der wichtigsten hämatologischen und onkologischen Therapieschemata nun mit den Steckbriefen zum häufig eingesetzten R-CHOP sowie hochdosierten Methotrexat (MTX). Mir liegt der richtige Umgang mit hochdosiertem Methotrexat persönlich besonders am Herzen, denn es ist eines der nebenwirkungsreichsten Therapieschemata, das die Hämatologie zu bieten hat, und der unsachgemäße Umgang hat möglicherweise verheerende Folgen für den Patienten. Insofern hoffe ich, dass Ihnen der Beitrag von Dr. Martin Janz vom Max-Delbrück-Centrum für Molekulare Medizin in Berlin den Umgang mit MTX ähnlich nahebringen kann, wie er ihn mir vor Jahren als erfahrener Stationskollege nahegebracht hat.

An immer größerer Bedeutung gewinnt jegliche Art von Simulationstraining in der Medizin, nicht nur für Notfallsituationen, sondern auch zum Erlangen kommunikativer Kompetenzen. Hier gibt Ihnen ein Beitrag zum Würzburger Schauspielpatienten-Programm entsprechende Einblicke. Aber auch im Bereich der Diagnostik gibt es interessante Entwicklungen. Bettina Otte von der Charité Berlin hat die Web-Anwendung NET-Xpert exklusiv für uns getestet.

In einem weiteren Beitrag nimmt der Medizinfachanwalt Dr. Thomas Ruppel Stellung zu rechtlichen Grundlagen von Patientenverfügungen, einem Thema, das sicher jeden von uns im Alltag gelegentlich nachdenken lässt.

Nun wünsche ich Ihnen viel Spaß beim Lesen und scheuen Sie sich nicht, uns bei Anregungen oder Kritik zu kontaktieren.

Herzliche Grüße,

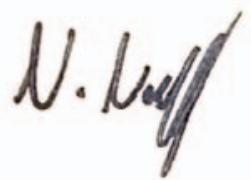

\section{KARGER}

Fax +497614520714 information@karger.com www.karger.com (c) 2017 S. Karger GmbH, Freiburg
Dr. med. Nina Rosa Neuendorff Medizinische Klinik V (Hämatologie,

Onkologie und Rheumatologie)

Universitätsklinik Heidelberg

Im Neuenheimer Feld 410 69120 Heidelberg 


\section{Indikation}

Vorbemerkung: R-CHOP ist die klassische «Standard»-Therapie bei B-Zell-Lymphomen mit höherer Wachstumsfraktion bzw. aggressivem biologischem Verhalten. Durch das Hinzufügen des AntiCD20-Antikörpers Rituximab wurde das Gesamtüberleben deutlich verbessert [1].

\section{Dosierung}

Prämedikation: Paracetamol 1000 mg p.o., Prednisolon 100 mg i.v., H1Antagonist (Dimetinden 4 mg i.v.), H2-Antagonist (Ranitidin 50 mg i.v.)

\begin{tabular}{llll} 
Rituximab & $375 \mathrm{mg} / \mathrm{m}^{2}$ i.v. & Tag 0 a & \\
\hline Cyclophosphamid & $750 \mathrm{mg} / \mathrm{m}^{2}$ & i.v. über $1 \mathrm{~h}$ & Tag 1 \\
\hline Doxorubicin & $50 \mathrm{mg} / \mathrm{m}^{2}$ & $\begin{array}{l}\text { i.v. über } 15 \\
\text { min }\end{array}$ & Tag 1 \\
\hline Vincristin & $1,4 \mathrm{mg} / \mathrm{m}^{2}$, max. $2 \mathrm{mg}^{\text {c }}$ & i.v. Bolus & Tag 1 \\
\hline Prednison & $100 \mathrm{mg}$ absolut & p.o. & Tag 1-5
\end{tabular}

a Bei Erstgabe: Start mit 50 mg/h, bei guter Verträglichkeit alle 30 min um 50 mg/h steigern bis max. 400 mg/h; engmaschige Überwachung des Patienten.

${ }^{\mathrm{b}}$ Cave: eine schnelle Injektion mit hohen Spitzenkonzentrationen scheint das Risiko der Kardiotoxizität zu fördern.

c Bei Patienten $\geq 70$ Jahre max. 1 mg.

\section{Begleitmedikation}

- Antiemese: moderat emetogenes Potential; 5- $\mathrm{HT}_{3}$-Rezeptor-Antagonist (z.B. Ondansetron $8 \mathrm{mg}$ als KI oder p.o. $3 \times$ an Tag 1)

- Hydrierung: 1 I NaCl 0,9\% + ggf. Kalium

- Mesna-Prophylaxe: bei der Biotransformation von Cyclophosphamid entsteht durch Abspaltung Acrolein, das urotoxisch wirkt (hämorrhagische Zystitis); Mesna bildet mit Acrolein ein nicht-toxisches, renal ausscheidbares Additionsprodukt; Dosierung: als Faustregel gilt 20\% der Cyclophosphamid-Dosis zu den Zeitpunkten Stunde 0, 4 und 8 i.v. oder p.o.

\section{Prophylaxen}

- Gabe von G-CSF: R-CHOP-14 mit G-CSF ab Tag 4, R-CHOP-21 nach zu erwartendem Infektionsrisiko

- Antiinfektive Prophylaxe:

1) antimikrobielle Prophylaxe: nicht routinemäßig empfohlen

2) Prophylaxe der Pneumocystis jirovecii-Pneumonie: Trimethoprim/Sulfamethoxazol (TMP/SMX), z.B. 960 mg 1 ×/Tag Mo-Mi-Fr. Die AGIHO empfiehlt die Prophylaxe mit TMP/ SMX für die Dauer der Therapie mit niedriger Evidenz [2]

3) Antivirale Prophylaxe gegen humane Herpesviren: Aciclovir $4 \times 200$ mg/Tag p.o.; keine generelle Empfehlung zur Prophylaxe

4) Prophylaxe einer Hepatitis-B-Reaktivierung: signifikantes Risiko unter Rituximab bis hin zum akuten Leberversagen; routinemäßiges Screening aller Patienten vor einer Therapie mit Rituximab ( $\mathrm{HBsAg}$, Anti-HBc); bei Positivität: Bestimmung der HBV-DNA; besondere Vorsicht bei HBsAg-positiven Patienten; auch bei durchgemachter HBV-Infektion (HBsAg negativ, Anti-HBcAg positiv) besteht die Möglichkeit der Reaktivierung; Prophylaxe mit Lamivudin oder Entecavir unter Therapie und bis zu 6-12 Monaten nach Therapie; geringere Resistenzentwicklung unter Entecavir [3]

5) Amphotericin-B-Suspension zur Vermeidung von lokalen Pilzinfektionen: Mundspülung zumindest für die Phase der Neutropenie und/oder bei Schleimhautschädigung, 4-6mal täglich, vor allem nach dem Essen

Zyklusintervall: 14-21 Tage

Merke: bei hoher Tumorlast Gefahr der Tumorlyse, daher ggf. Vorphase-Therapie einleiten, insb. bei älteren Patienten und/oder hoher Tumorlast (Prednisolon $100 \mathrm{mg}$ absolut p.o./Tag über ca. 7 Tage, ggf. plus Vincristin 1 mg absolut i.v.)

\section{Dosismodifikationen}

- Dosisbegrenzung von Vincristin bei älteren Patienten ( $\geq 70$ Jahre) auf max. $1 \mathrm{mg}$ absolut.

- Dosisreduktion von Vincristin bei Zeichen einer Polyneuropathie.

- Kardiotoxizität von Anthrazyklinen beachten! Für Doxorubicin wird als gefährliche kumulative Dosis $550 \mathrm{mg} / \mathrm{m}^{2}$ angegeben [4], allerdings ohne exakt definierten Schwellenwert. Die Kardiotoxizität kann sich akut (Rhythmusstörungen) oder noch Monate bis Jahre nach Applikation manifestieren (Herzinsuffizienz); Häufigkeit vermutlich unterschätzt.

\section{Generelle Untersuchungen vor Einleitung der Therapie}

- EKG, transthorakale Echokardiographie, auf klinische Zeichen einer Herzinsuffizienz achten

- nach Symptomen einer Polyneuropathie fragen (Kribbelparästhesien, Taubheitsgefühl, Probleme beim Schreiben oder Knöpfe schließen)

- Lungenfunktion (initial zur Abschätzung des pulmonalen Risikos, weniger durch eine direkte Toxizität als vielmehr durch Infektionen, z.B. bei COPD)

\section{Kontraindikationen}

Relative oder absolute Kontraindikationen ergeben sich vor allem durch die wichtigsten Organtoxizitäten: Kardiotoxizität durch Doxorubicin und Polyneuropathie durch Vincristin - entscheidend ist, dies durchweg im Blick zu behalten! 
Steckbriefe der wichtigsten Chemotherapieschemata

\section{R-CHOP}

\section{? Wichtige Fragen}

\section{Was muss vor jeder Gabe der Therapie körperlich unter- sucht werden?}

Adäquater Allgemeinzustand? Infektzeichen? Hinweise auf eine kardiale Schädigung? Symptome einer Polyneuropathie?

Wie oft müssen Laborwerte während der Therapie ermittelt werden und wie oft muss der Patient untersucht werden?

- mind. wöchentliche Blutbildkontrollen, insb. um die Tiefe des Nadirs abschätzen zu können (wichtig um Tag 10-12); ggf. Blutbildkontrolle bei Fieber (Gefahr der Neutropenie)

- vor Beginn eines Zyklus: Blutbild (ausreichende hämatologische Rekonstitution? Als orientierende Grenzwerte gelten

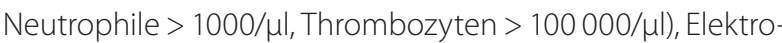
lyte, Kreatinin, Harnsäure, Leberwerte, Gerinnung

\section{Notwendigkeit eines zentralen Zugangs?}

Bei guten Venenverhältnissen nicht unbedingt erforderlich, aber äußerste Vorsicht bei der Applikation über einen peripheren Zugang (Doxorubicin und Vincristin haben starke gewebsschädigende Wirkungen! Paravasatgefahr!). In der Praxis wird daher sehr häufig ein Port gelegt, um die Sicherheit zu erhöhen.

\section{Bei welchen Komplikationen muss sich der Patient drin- gend ärztlich vorstellen?}

Die bei weitem größte Gefahr stellt Fieber dar. In der Neutropenie kann dies lebensgefährlich werden!

\section{IIIl Für den Notfall}

\section{Nebenwirkungen}

- bei erstem Zyklus und hoher Tumorlast: Gefahr der Tumorlyse (genaue Überwachung der Elektrolyte einschließlich Calcium und Phosphat, Kreatinin, Harnsäure, LDH, Gerinnung)

- generell: Gefahr der Hämatotoxizität mit erhöhtem Infektionsrisiko, Nadir zwischen Tag 10 und 12, Fieber in der Neutropenie, Blutungen

\section{Notfallmaßnahmen bei Paravasat}

Achtung: Anthrazykline und Vinca-Alkaloide sind stark gewebsschädigende, nekrotisierende Substanzen!

Sofortmaßnahmen bei Paravasat:

- Infusion stoppen

- Flexüle oder Portnadel zunächst belassen

- Paravasat-Set holen, Handschuhe anziehen

- Infusionsleitung entfernen und Spritze anschließen; Versuch einer vorsichtigen Aspiration

- Flexüle oder Portnadel unter vorsichtiger Aspiration entfernen

- betroffene Extremität hochlagern und ruhigstellen
- sorgfältige Kontrollen und Dokumentation des Lokalbefunds - spezifische Maßnahmen (s.a. S3-Leitlinie «Supportive Therapie» [5]):

1) Anthrazykline:

a) i.v. Gabe von Dexrazoxan (Savene ${ }^{\circledR}$ ): möglichst frühe Applikation (in den ersten 6 h), Behandlung über 3 Tage (1. Tag: $1000 \mathrm{mg} / \mathrm{m}^{2} ; 2$. Tag: $1000 \mathrm{mg} / \mathrm{m}^{2}$; 3. Tag: $500 \mathrm{mg} / \mathrm{m}^{2}$; Vorsicht bei Niereninsuffizienz)

b) wenn keine i.v. Behandlung, dann lokale Applikation von DMSO alle 8 h für 7 Tage; betupfen, kein Druck, kein Reiben, keine Abdeckung (DMSO soll an der Luft trocknen); bei gleichzeitiger lokaler Therapie mit Kälte Intervall von mind. 15 min einhalten

2) Vinca-Alkaloide: Paravasatstelle intradermal oder subkutan periläsional mit Hyaluronidase umspritzen (Dosierung: 1-10 Ampullen à $150 \mathrm{IU}$, Angaben schwanken in der Literatur zwischen 100 bis zu 1500 IU, Lösung in $1 \mathrm{ml}$ Lösungsmittel, z.B. NaCl 0,9\%; lokale Analgesie, z.B. mit Lidocain 1\%)
Literatur

1 Coiffier B, Lepage E, Briere J, et al.: CHOP chemotherapy plus rituximab compared with $\mathrm{CHOP}$ alone in elderly patients with diffuse large-B-cell lymphoma. $\mathrm{N}$ Engl J Med 2002;346:235-242.

2 Neumann S, Krause SW, Maschmeyer G, et al.: Primary prophylaxis of bacterial infections and Pneumocystis jirovecii pneumonia in patients with hematological malignancies and solid tumors: guidelines of the Infectious Diseases Working Party (AGIHO) of the German Society of Hematology and Oncology (DGHO). Ann Hematol 2013;92:433-442.

3 Huang H, Li X, Zhu J, et al.: Entecavir vs lamivudine for prevention of hepatitis B virus reactivation among patients with untreated diffuse large B-cell lymphoma receiving R-CHOP chemotherapy: a randomized clinical trial. JAMA 2014;312: 2521-2530.
4 Von Hoff DD, Layard MW, Basa P, et al.: Risk factors for doxorubicin-induced congestive heart failure. Ann Intern Med 1979;91:710-717.

5 Leitlinienprogramm Onkologie: S3-Leitlinie Supportive Therapie bei onkologischen PatientInnen. www.leitlinienprogramm-onkologie.de/leitlinien/supportivetherapie (letzter Aufruf: 15.09.2017).

Kontaktadresse: PD Dr. Martin Janz, Hämatologie/Onkologie, Charité, Campus Benjamin Franklin, Hindenburgdamm 30, 12200 Berlin, Deutschland, martin.janz@charite.de. 


\section{Definition}

HD-MTX $\geq 500 \mathrm{mg} / \mathrm{m}^{2}$ i.v.

\section{Indikation}

- akute lymphatische Leukämie (ALL) und hochmaligne NonHodgkin-Lymphome (z.B. Burkitt-Lymphom)

- ZNS-Prophylaxe bei Lymphomen, primäre ZNS-Lymphome

- Osteosarkom, Medulloblastom

Merke: sehr gute Penetration in das ZNS!

\section{Wirkungsmechanismus}

Wirkung als Antimetabolit/Folsäure-Antagonist; kompetitive und reversible Inhibition der Dihydrofolatreductase (DHFR), daher durch Gabe von Folinsäure (Leucovorin ${ }^{\circledR}$ ) antagonisierbar (sog. Leucovorin-Rescue)

\section{Dosierung}

- $0,5-12 \mathrm{~g} / \mathrm{m}^{2}$

- es existieren vor allem 2 Schemata:

a) schnelle Gabe über $4 \mathrm{~h}$ mit hohem MTX-Spitzenspiegel

b) protrahierte Gabe über $24 \mathrm{~h}$ mit längerer MTX-Exposition

\section{Grundlagen}

MTX wird nach i.v. Gabe zu 80-90\% unverändert durch glomeruläre Filtration und tubuläre Sekretion über die Nieren ausgeschieden [1]. MTX ist eine Säure, die aktiv über entsprechende Transporter-Systeme im proximalen Tubulusapparat in den Harn ausgeschieden wird (z.B. via organic anion transporter (OAT) 1/3 [2]). Bei allen sauren Pharmaka besteht daher prinzipiell die Gefahr, dass die Sekretion von MTX behindert wird - mit unter Umständen fatalen Folgen! Vorsicht vor Interaktionen (siehe unten)! Volumendepletion und saurer Urin sind weitere wesentliche Risikofaktoren für ein akutes Nierenversagen; Ursache ist eine mögliche Präzipitation von MTX in den Tubuli (geringe Löslichkeit von MTX bei saurem pH-Wert).

Bei pathologischen Flüssigkeitsansammlungen wie z.B. Aszites, Pleuraerguss besteht ein sogenannter 3. Raum, aus dem MTX nur langsam zurückflutet, sodass die MTX-Elimination verzögert und die toxische Wirkung verlängert wird; ggf. vorher sorgfältige Punktion/Drainage.

\section{Durchführung}

Allgemeine Vorbemerkung: Aus organisatorischen Gründen hat es sich in vielen Kliniken etabliert, exakt um 14:00 Uhr mit der MTXInfusion zu beginnen. Dies ermöglicht ein absolut standardisiertes Vorgehen in Bezug auf Start, Blutentnahmen und Beginn des Leucovorin-Rescue, wodurch Versäumnisse und Fehler reduziert werden können.

\section{Medikamenteninteraktionen beachten}

Je leerer die Kurve desto besser! Nur wirklich notwendige Medikamente ohne erkennbares Interaktionspotential belassen! Im Zweifel - wenn möglich - immer pausieren!

Beispiele:

- NSAR, Salicylate, Penicillin und Penicillin-Derivate, Probenicid, Trimethoprim-Sulfamethoxazol, Ciprofloxacin, Protonenpumpen-Inhibitoren u.v.a.

- den Urin ansäuernde Getränke (Fruchtsäfte, Cola, Vitamin C)

- potentiell nephrotoxische Substanzen vermeiden (z.B. Kontrastmittel, Aminoglykoside, Amphotericin)

\section{Volumengabe und Harnalkalisierung}

- Hydratation: am Vortag beginnen, ca. 150-200 ml/h (gesamt pro Tag 3-5 I); Achtung bei eingeschränkter kardialer Funktion; Bilanzierung bzw. Gewicht mindestens $2 \times /$ Tag bestimmen, ggf. mit Furosemid gegensteuern

- Alkalisierung des Urins: Messung des Urin-ph-Werts bei jeder Miktion; Ziel: pH > 7; ggf. Na-Bikarbonat i.v., orale Gaben sind nur mäßig und langsamer wirksam, jedoch in den meisten Protokollen als Basismedikation enthalten; Werte $\mathrm{pH}>7$ müssen bereits vor dem Start erreicht und dokumentiert werden!

\section{MTX-Spiegel und Leucovorin-Rescue}

- den Abfall des MTX-Spiegels genau verfolgen (detaillierte Anleitungen in den Therapie-Protokollen)

- pünktlicher Start der Gabe von Folinsäure i. v. (Leucovorin-Rescue); je nach Schema (4 h vs. 24 h) Beginn zu Stunde 24 bzw. 42; Dosisanpassung je nach MTX-Spiegelverlauf; Fortsetzen bis sichere Werte erreicht werden (was als sicher erachtet wird, variiert zwischen Protokollen und klinischer Situation; Richtwerte $<0,1$ $\mu \mathrm{mol} / \mathrm{l}$ bis $<0,04 \mu \mathrm{mol} / \mathrm{l}$ )

- mindestens tägliche Kontrollen von Elektrolyten, Kreatinin, Leberwerten

- unzureichend abfallende MTX-Spiegel sind immer ein Grund, einen Fach- oder Oberarzt zu Rate zu ziehen - auch nachts! Im Notfall bei unzureichendem Abfall des MTX-Spiegels und sich abzeichnender starker Toxizität wie akutes Nierenversagen (ANV) oder neurologischen Komplikationen: Gabe von Carboxypeptidase G2 (Glucarpidase, Voraxaze ${ }^{\circledR}$ ), senkt die MTX-Spiegel durch enzymatischen Abbau [3], direkter Bezug über Clinigen (wertvolle Informationen zum Bezug s. [4]). 


\section{? Wichtige Fragen}

Was muss vor jeder Gabe der Therapie körperlich untersucht werden?

Adäquater Allgemeinzustand? Infektzeichen? Mukositiszeichen? Volumenzustand? 3. Raum (Aszites, Pleuraerguss)?

\section{Notwendigkeit eines zentralen Zugangs?}

Aufgrund der hohen Volumengabe, der eventuellen Notwendigkeit von Bikarbonatgaben sowie den sehr häufigen Blutentnahmen ist ein zentraler Zugang dringend zu empfehlen.
Bei welchen Komplikationen muss sich der Patient dringend ärztlich vorstellen?

Mukositis; Fieber: dies kann insbesondere bei kombinierten Protokollen (z.B. ALL-Behandlung) sehr ausgeprägt sein und die Form eines lebensbedrohlichen Fiebers in der Neutropenie verschärft durch die Schleimhautschädigung und die damit einhergehende Keimeinschwemmung - annehmen.

\section{Für den Notfall}

\section{Toxizitäten/Kontraindikationen}

Merke: Als Akut-Toxizitäten stehen vor allem Nephrotoxizität und Mukositis, gefolgt von Hepatotoxizität und weniger ausgeprägt Hämatotoxizität im Zentrum.

Merke: Die Applikation von MTX ohne adäquates Rescue ist potenziell tödlich!

- Nephrotoxizität: wichtigste akute Toxizität bis hin zum schweren ANV mit Dialysepflicht; besonders achten auf mögliche Medikamenteninteraktionen, Volumenstatus vor Therapiestart, Urin-pH (Ziel pH > 7), zusätzliche Risikofaktoren (bestehende Nierenfunktionseinschränkung, Diabetes, arterielle Hypertonie usw.); bei vorangegangenem Nierenversagen sorgfältig die Indikation prüfen

- Mukositis: Mund/Rachenraum, aber auch gastrointestinale und anale Läsionen
- Hepatotoxizität: häufig passagerer Transaminasenanstieg, bleibende Leberveränderungen wie Fibrose oder Zirrhose möglich

- Hämatotoxizität: möglich, besonders bei protrahierten Spiegelabfällen

- Neurotoxizität: Kopfschmerzen, Benommenheit, Sehstörungen, Verhaltensauffälligkeiten, Krampfanfällen, fokale sensomotorische Symptome bis zur akuten toxischen Leukenzephalopathie; Verstärkung durch vorangegangene ZNS-Bestrahlung

- Lungentoxizität: Alveolitis, Pneumonitis

- gering emetogen

- gelegentlich Alopezie

\section{Literatur}

1 Shen DD, Azarnoff DL: Clinical pharmacokinetics of methotrexate. Clin Pharmacokinet 1978;3:1-13.

2 Hagos Y, Wolff NA: Assessment of the role of renal organic anion transporters in drug-induced nephrotoxicity. Toxins (Basel) 2010;2:2055-2082.

3 Schwartz S, Borner K, Müller K, et al.: Glucarpidase (carboxypeptidase g2) intervention in adult and elderly cancer patients with renal dysfunction and delayed methotrexate elimination after high-dose methotrexate therapy. Oncologist 2007;12 $1299-1308$
4 Schwartz S, Fleischhack G, Grüneberg I: Methotrexat (MTX) - Toxischer Spiegelverlauf. www.kinderkrebsinfo.de/fachinformationen/notfallempfehlungen/methotrexat/index_ ger.html (letzter Abruf: 15.09.2017).

Kontaktadresse: PD Dr. Martin Janz, Hämatologie/Onkologie, Charité, Campus Benjamin Franklin, Hindenburgdamm 30, 12200 Berlin, Deutschland, martin.janz@charite.de. 
Arzt-Patienten-Kommunikation

\section{Schauspielpatienten-Programm an der Medizinischen Fakultät der Universität Würzburg}

Krankheitsverlauf, Adhärenz, Behandlungserfolg und nicht zuletzt eine gelungene Kommunikation [1] sind Aspekte, die eine gute ArztPatienten-Beziehung positiv beeinflussen. Um den Grundstein für diese essenziellen kommunikativen Fähigkeiten möglichst bereits im Medizinstudium zu legen, werden weltweit an zahlreichen Universitäten Schauspielpatienten eingesetzt, d.h. Laienschauspieler, die für bestimmte Patientenrollen trainiert werden: Dies umfasst nicht nur eine medizinische Vorgeschichte mit Symptomen, Voruntersuchungen usw., sondern auch nicht-medizinische Informationen wie den Beruf oder die familiäre Situation des erdachten Patienten. Ziel ist es, einen möglichst authentischen Patienten entstehen zu lassen. Seit 2007 gibt es das Schauspielpatienten-Programm an der Universität Würzburg. Wir hatten die Möglichkeit, mit Dr. Matthias Lukasczik und Dipl.Psych. Hans-Dieter Wolf, den beiden Koordinatoren des Programms, zu sprechen.

\section{Wie läuft ein Schauspielpatientengespräch ab?}

Die Gespräche finden in Kleingruppen von maximal 12 Personen statt und dauern jeweils ca. 10 min. Sie werden in den Lehrveranstaltungen vorbereitet, d.h. die Dozenten erarbeiten mit den Studenten allgemeine Informationen und geben Input zur Situation und zu den Fertigkeiten, die für ein gutes Gespräch wichtig sind. Außerdem erhalten die Studenten eine schriftliche Kurzinformation mit Angaben zum Patienten, zu medizinischen Fakten und zu ihrer Aufgabe als Arzt im Gespräch.

Im Gespräch selbst sind jeweils ein Dozent bzw. Tutor sowie die anderen Mitglieder der Kleingruppe anwesend. Nach dem Gespräch folgt ein strukturiertes Feedback: Nachdem der Student, der die Arztrolle übernommen hat, seine Einschätzung des Gesprächs abgegeben hat, äußert sich der Schauspielpatient (SP) dazu, was im

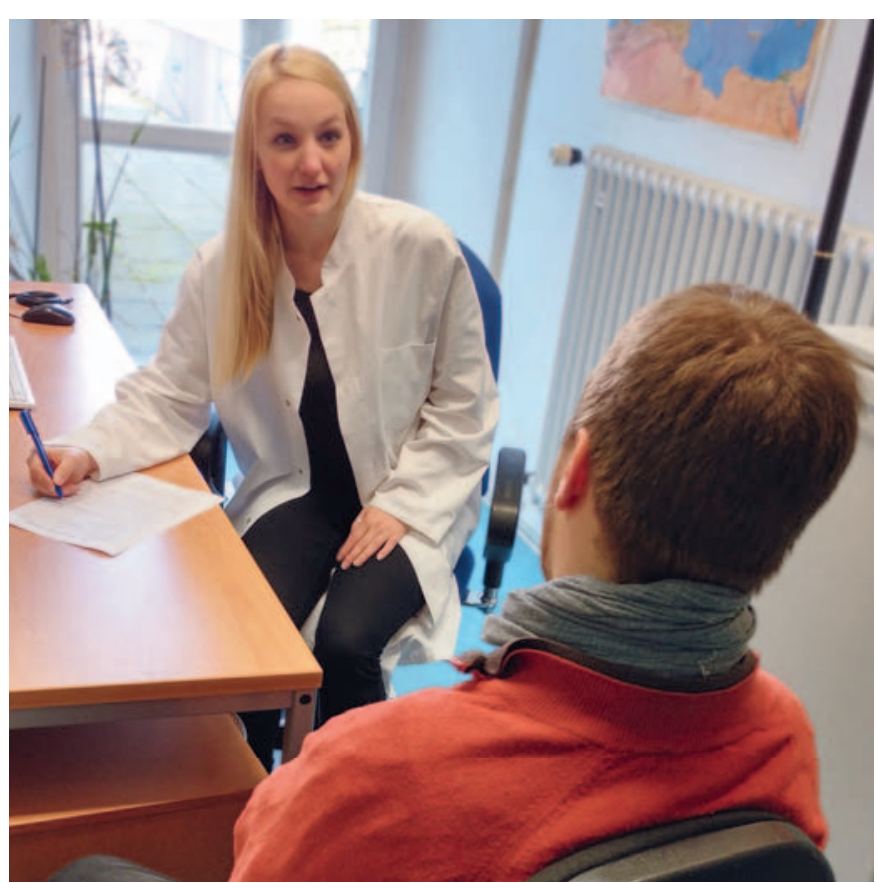

CMatthias Lukasczik, Schauspielpatienten-Programm Würzburg.
Gespräch gut gelaufen ist und an welchen Stellen es Verbesserungsbedarf gibt. Danach geben der Dozent und die anderen Kursteilnehmer Rückmeldung.

\section{Wie wird eine Patientenrolle kreiert?}

Zunächst überlegen wir uns, wo Bedarffür eine neue Rolle besteht. Hieraus leiten sich in der Regel schon einige Vorgaben ab (Erkrankung, Lebensstil des Patienten, vermutliche Gefühlslage usw.).

Mithilfe dieses Grundgerüstes folgt in der Regel zuerst eine Art Brainstorming mit den für das jeweilige Fach verantwortlichen Dozenten. An der detaillierten Formulierung der Rolle sind immer mehrere Personen aus verschiedenen Berufsgruppen (Psychologen, Ärzte) beteiligt.

Die Rolle umfasst im Ergebnis dann mindestens die folgenden Informationen:

- Angaben zur Person (Name, Alter inkl. Geburtsdatum, Familie)

- Angaben zur beruflichen Situation und Laufbahn

- Angaben zur aktuellen medizinischen Situation

- Angaben zur medizinischen Vorgeschichte

- Angaben zu Persönlichkeit und sozialer Situation

Die so entstandene Rolle wird dann mit einem oder mehreren SP durchgearbeitet und im Rollentraining praktisch getestet. Input von Schauspielerseite wird aufgegriffen, mögliche Unklarheiten oder «Stolpersteine» werden berücksichtigt und die Rolle entsprechend angepasst. Erst dann kann sie in der Lehrroutine eingesetzt werden.

Gibt es «schwierige» Rollen, die beispielsweise den kulturellen Hintergrund und die damit verbundenen Herausforderungen berücksichtigen?

Es gibt Rollen, die aufgrund ihrer medizinischen Situation herausfordernd sind - dazu zählt z.B. die Rolle einer jungen Mutter mit fortgeschrittener Krebserkrankung, die aller Wahrscheinlichkeit nach ihr Kind nicht aufwachsen sehen wird. Andere sicher sinnvolle und spannende Konstellationen, z.B. Angehörigengespräche oder Rollen aus anderen kulturellen Hintergründen, sind Teil unserer langfristigen Planungen, bislang aber noch nicht umgesetzt.

Welche Problemfelder/Herausforderungen haben sich im Laufe der Jahre herauskristallisiert und was kann man daraus für die Praxis lernen?

Eine der größten Schwierigkeiten, mit der gerade junge Mediziner zurechtkommen müssen, ist der immer größer werdende Zeitdruck im ärztlichen Berufsalltag. Eine der großen Herausforderungen ist es daher, zu zeigen, dass man auch in zehn Minuten eine gute Beziehung zu einem Patienten aufbauen und auf seine Bedürfnisse eingehen kann.

Aufgrund der demographischen Entwicklung nimmt auch die Arbeit mit älteren Menschen einen immer größeren Raum in der ärztlichen Arbeit ein. Deshalb sind mehrere Rollen im Würzburger SP- 
Programm bereits auf ältere Patienten zugeschnitten, um den Studierenden das Üben alltagsrelevanter Gesprächssituationen zu ermöglichen.

Welche Entwicklungen gab es in den nunmehr 10 Jahren, in denen das Programm schon läuft und welche Pläne gibt es für die Weiterentwicklung des Programms?

In erster Linie ist das Programm ausgebaut und erweitert worden - sowohl was den Pool an Rollen als auch was die Zahl der Lehrveranstaltungen mit SP-Einsätzen betrifft. Mittlerweile ist die Arbeit mit SP ein fester Bestandteil der medizinischen Lehre in Würzburg und wird von den Studenten sehr gut bewertet. Der hohe Zuspruch zeigt sich auch an den vielen Interessierten, die sich als Tutoren bzw. als SP bewerben. Die gute Entwicklung des Programms findet fächerübergreifend Anerkennung und wurde 2011 mit dem AlbertKölliker-Lehrpreis der Medizinischen Fakultät ausgezeichnet.

Ein Faktor, der für die medizinische Ausbildung sicher relevant ist, ist der Nationale Kompetenzbasierte Lernzielkatalog Medizin (NKLM). Dort wird Kommunikationsfertigkeiten ein großer Stellenwert beigemessen. Daher wird sicher die Frage nach der Prüfbarkeit der erlernten Kommunikationskompetenzen, z.B. im Rahmen von OSCE-Prüfungen, noch mehr in den Vordergrund rücken. Hier steht das SP-Programm in Würzburg noch in der Planungsphase.

Wir danken Ihnen herzlich für das Interivew!

\section{Angaben zum Schauspielpatienten-Programm in Würzburg}

Das Programm wurde 2007 von Ärzten und Psychologen des Uniklinikums (Palliativmedizin) und der Universität (Medizinische Psychologie) initiiert, damals noch beschränkt auf den Bereich Onkologie. Aktuell werden Schauspielpatienten in 4 Lehrveranstaltungen eingesetzt: «Medizinische Psychologie» (Anamnese, Motivierung zur Lebensstiländerung, Aufklärung/ Diagnosemitteilung), «Praktische Klinische Untersuchungsmethoden» (Anamnese), «Kommunikationstraining Interdisziplinäre Onkologie» (Diagnosemitteilung) und «OP-Aufklärung». Insgesamt kommen derzeit ca. 30 Schauspielerinnen und Schauspieler in 20 verschiedenen Rollen zum Einsatz.

Nähere Informationen finden Sie unter www.psychotherapie.uni-wuerzburg.de/schauspielpatienten

\author{
Literatur \\ 1 Interview mit Samy Molcho: Von Mensch zu Mensch statt von Arzt zu Patient. \\ Karger Kompass Dermatol 2017:5;98.
}

Kontaktadresse: Dr. Matthias Lukasczik / Dipl.-Psych. Hans-Dieter Wolf, JuliusMaximilians-Universität Würzburg, Abteilung für Medizinische Psychologie, Klinikstraße 3, 97070 Würzburg, Deutschland, matthias.lukasczik@ uniwuerzburg.de; hans.wolf@uni-wuerzburg.de.

\section{Medizinrecht \\ Patientenverfügungen - Was müssen junge Ärzte beachten?}

Kassenarztrecht, Praxisrecht, Vertragsgestaltung, ärztliches Arbeitsrecht, Medizinstrafrecht und ärztliches Berufsrecht sind nur einige Stichpunkte aus dem Medizinrecht. Rechtsanwalt Dr. Ruppel aus Lübeck gibt Einblicke zu rechtlichen Fragen und zur Selbstständigkeit als Arzt.

\section{Jede ärztliche Maßnahme ist einwilligungsbedürftig}

Jede, auch noch so gut gemeinte, ärztliche Behandlung ist eine straf- und zivilrechtlich geahndete Körperverletzung: Sie stellt ohne Einwilligung des Patienten eine Straftat dar, außerdem stehen Patienten Unterlassung-, Schadensersatz- und Schmerzensgeldansprüche zu.

Bei volljährigen, bei Bewusstsein befindlichen Patienten erfolgt die Rechtfertigung dieser aus rechtlicher Sicht eigentlich zu sanktionierenden ärztlichen Eingriffe durch eine Einwilligung. Deshalb ist auch die umfassende und rechtzeitige Aufklärung so wichtig - nur so kann eine wirksame Einwilligung erreicht werden.

Kann der Patient die Einwilligung nicht geben, so ist sein mutmaßlicher Wille zu ermitteln: Was hätte der Patient gewollt, hätte man inn fragen können?

\section{Patientenverfügung als Möglichkeit, den Willen des Patienten zu vermitteln}

Ein mittlerweile verbreitetes Instrument hierfür ist die Patientenverfügung. Sie soll, wenn sie gut abgefasst ist, den Willen des Patienten ermittelbar machen. Jeder Arzt ist verpflichtet, die Patientenverfügung zu beachten, wenn sie wirksam ist. Zu ihrer Wirksamkeit muss sie schriftlich verfasst worden sein, mittels Computer reicht aus. Sie sollte aber zumindest unterschrieben sein. Auch muss der Patient bei Abfassen der Patientenverfügung noch begriffen haben, was er verfügt.

Die inhaltliche Wirkung einer Patientenverfügung ergibt sich oftmals aus dem Wortlaut. Dieser ist stets ein wichtiges Indiz zu ihrer Auslegung. Jedoch darf man nicht am Wortlaut kleben bleiben. Entscheidend ist immer der Wille des Patienten, wie er sich aus dem Gesamtkontext der Patientenverfügung und ggf. auch durch Befragung der Angehörigen ergibt, selbst wenn er vom Wortlaut abweicht.

Wenn ein Patient etwa schreibt, er möchte nicht an «Maschinen angeschlossen» werden, so wollen die allermeisten Patienten durchaus auch eine Schmerztherapie, die ggf. den «Anschluss» an eine Maschine notwendig macht. Gleiches gilt etwa, wenn eine Beatmung abgelehnt wird - die meisten Patienten mit Atemproblemen dürften sich damit gegen eine Intubation entscheiden, jedoch eine Unterstützung durch eine nicht-invasive Beatmung wünschen. Wenn der Patient einen Organspendeausweis ausgefüllt hat und die dort niedergelegte Entscheidung immer noch seinem Willen entspricht, dann haben die Angehörigen kein Mitspracherecht über die Entscheidung zur Organentnahme. Der durch die Auslegung ermittelte Wille des Patienten ist umzusetzen. 


\section{Unwirksame Inhalte von Patientenverfügungen}

Manche in Patientenverfügungen geäußerten Wünsche sind jedoch unzulässig oder nicht umsetzbar: Bekanntlich ist in Deutschland die aktive Sterbehilfe verboten. Dem Verlangen aus einer Patientenverfügung oder von Angehörigen aktive Sterbehilfe zu leisten, ist nicht nachzukommen. Erlaubt sind hingegen die indirekte und die passive Sterbehilfe, d.h. das Sterbenlassen durch Abschalten von lebenserhaltenden Geräten sowie die Durchführung von Therapien, die als Nebenwirkung zu einem verfrühten Tod des Patienten führen, etwa bei hoch dosierten Schmerzmitteltherapien. In der Praxis untauglich und von der Rechtsprechung mittlerweile auch für unwirksam erachtet, sind Allgemeinsätze wie der Wunsch des Patienten, «in Würde zu sterben» oder ein «menschenwürdiges Leben zu führen». Niemand weiß, was der Patient hiermit wirklich sagen wollte - und kein Arzt möchte einem Patienten einen würdevollen Tod vorenthalten.

In der Krankenhauspraxis untauglich sind viele Patientenverfügungen zum Ankreuzen, weil sie kausale «wenn-dann-Abläufe» voraussetzen, die in den allermeisten Fällen nicht gegeben sind. Insbesondere bei zum Zeitpunkt des Aufsetzens der Patientenverfügung noch mehr oder weniger gesunden Patienten und bei multimorbiden Patienten, treten die von den Ankreuz-Patientenverfügungen vorausgesetzten Kausalverläufe nicht ein. Dies ist, wenn überhaupt, nur bei austherapierten Patienten mit bestimmten Krankheiten vorstellbar. Wir empfehlen sogenannte «Wunsch-Werte-Angst»Profile, d.h. nicht die Entscheidung des Patienten über einzelne, von im Zweifel überhaupt nicht zu überblickende medizinische Maßnahmen, sondern eine detaillierte «Anamnese» des eigenen Lebens, der eigenen Wünsche und der eigenen Ängste. Auf diese Weise kann in jeglichen Situationen der mutmaßliche Wille des Patienten wirklich ermittelt werden.

\section{Patienten mit Vorsorgevollmachten}

Juristisch einfach ist die Entscheidung, wenn eine Vorsorgevollmacht als Generalvollmacht besteht, die einem Angehörigen oder Freund auch die Möglichkeit einräumt, in medizinischen Inhalten zu entscheiden. Viele Vorsorgevollmachten beinhalten diese Erlaubnis jedoch gerade nicht, sondern räumen eine Vertretungsmacht in Pflegeangelegenheiten ein. Hier müssen Sie die Vorsorgevollmacht sorgsam lesen.

Selbst wenn die rechtlichen Fragen damit gelöst sind, sind solche Vorsorgevollmachten oftmals inhaltlich wenig hilfreich: Denn welchen Willen der Patient gehabt hätte, lässt sich aus einer Vorsorgevollmacht, die nur einem Dritten das Recht zu entscheiden einräumt, nicht herauslesen. Deshalb ist es im Gespräch mit dem Vorsorgebevollmächtigten unerlässlich, auch hier zwischen dem Willen des Bevollmächtigten und des Vollmachtgebers (d.h. des Patienten) streng zu unterscheiden.

\section{Vorgehen bei unwirksamen oder fehlenden \\ Patientenverfügungen}

Besteht keine Patientenverfügung, ist diese unwirksam oder unklar, kann der mutmaßliche Wille des Patienten auch durch die Befragung von Angehörigen oder Freunden herausgefunden

werden. Dabei ist jedoch stets zu beachten, dass die von den Interessen des Patienten möglicherweise abweichenden Interessen der Angehörigen sorgfältig zu unterscheiden sind. Es kommt nicht auf das Interesse der Angehörigen an, sondern auf das des Patienten.

Besteht keine wirksame Patientenverfügung und hat der Arzt den Eindruck, dass die Angehörigen eher den eigenen Willen als den des Patienten formulieren, so empfiehlt es sich, den Betreuungsrichter am Amtsgericht zu informieren und eine Betreuung (früher: Vormundschaft) des Patienten anzuregen. Der Richter kommt dann in das Krankenhaus. Liegen die gesetzlichen Voraussetzungen vor, entscheidet der dann bestellte Betreuer über den Fortgang und den Abbruch von medizinischen Maßnahmen.

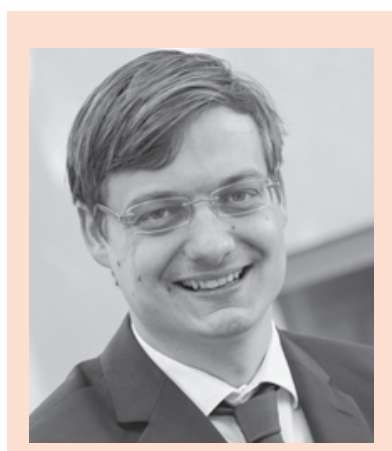

Dr. Thomas Ruppel, Lübeck

\section{Zum Autor}

Dr. Thomas Ruppel ist Rechtsanwalt für Medizinrecht in Lübeck. Er studierte Rechtswissenschaften an der Universität Greifswald. Bereits während des Studiums richtete er seinen Fokus auf das Medizinrecht. Seinen juristischen Vorbereitungsdienst absolvierte er am Landgericht in Düsseldorf, bei einer Kassenärztlichen Vereinigung und mittelständischen medizinrechtlichen Kanzleien, die ausschließlich Ärzte und andere Leistungserbringer im Gesundheitswesen beraten. Dr. Ruppel promovierte im Bereich der Versorgungsforschung und war unter anderem für eine internationale Rechtsanwaltskanzlei im Bereich Medizinrecht, Arzneimittelrecht und Medizinprodukte tätig.

Im Jahr 2012 absolvierte Dr. Ruppel den Fachanwaltslehrgang für Medizinrecht. Er veröffentlicht regelmäßig in der juristischen und ärztlichen Fachpresse.

Kontaktadresse: Dr. Thomas Ruppel, Rechtsanwälte Dr. Ruppel, Eschenburgstraße 21, 23568 Lübeck, Deutschland,www.gesundheitsrecht.de, kanzlei@ gesundheitsrecht.de, Tel.: 0451/29366 500 .

\section{Originalquelle}

Patientenverfügungen - was müssen junge Ärzte wissen?, in Operation Karriere - Das Karriereportal und der Kongress für angehende und junge Ärzte. www.operationkarriere.de. (Direktlink für Online-Version: www.operation-karriere.de/karriereweg/ von-beruf-arzt/patientenverfuegungen-was-muessen-junge-aerzte-beachten.html)

\section{OPERATION}




\section{Simulationstraining für Ärzte}

\section{NET-Xpert - Das neue Online-Training zur Behandlung von neuroendokrinen Tumoren}

Gerade im Bereich der neuroendokrinen Tumoren (NET) besteht großer Bedarfan Weiterbildungsmöglichkeiten für Ärzte, da die Seltenheit und Heterogenität dieser Tumoren - seien sie gut- oder schlecht-differenziert, hoch- oder niedrig-proliferativ, funktionell aktiv oder inaktiv - die Behandlung zu einer komplexen Aufgabe machen. Novartis hat im Sommer 2017 in Zusammenarbeit mit Dr. Alexander König, Universitätsmedizin Göttingen, und PD Dr. Kia Homayounfar, RKH Kassel, eine Web-Anwendung veröffentlicht, welche den Umgang mit NET erleichtern soll. Auf der Internetseite www.net-xpert.leben-mit-net.de können Ärzte ihr Wissen über Diagnostik und Therapie an realen Patientenfällen überprüfen und vertiefen.

\section{Wie funktioniert NET-Xpert?}

Das Online-Training lässt sich ohne komplizierten Download oder Installation im Browser öffnen. Es erfordert nur das Einloggen mittels DocCheck-Zugang. Es öffnen sich 3 Patientenfälle, von denen aktuell einer zur Bearbeitung freigeschaltet ist. Die anderen beiden folgen in 2018. Bei dem ersten Fall handelt es sich um einen Patienten mit zufällig diagnostizierten Lebermetastasen, bei dem schließlich die Diagnose eines mittelgradig-differenzierten NET mit ilealem Primarius gestellt wird. Durch Patientenfotos und ausführliche Anamnese entsteht ein hoher Realitätsbezug. Schrittweise wird man von dem Programm durch bar - in einer virtuellen Patientenakte abgelegt. Der Benutzer erhält unmittelbar Feedback zu der eigenen Therapieentscheidung. Ausführliche Kommentare erklären, warum die aktuelle Wahl sinnvoll oder vielleicht weniger sinnvoll ist. Nach abgeschlossener Diagnostik wird man in gleicher Weise durch die verschiedenen Therapieoptionen geführt, in diesem Fall die Primärtumor-Resektion mit anschließender Somatostatin-Analoga-Therapie.

\section{Fazit}

Novartis hat mit NET-Xpert ein sehr praxisnahes und gut verständliches Tool entwickelt, welches Potenzial hat, die Kenntnisse über

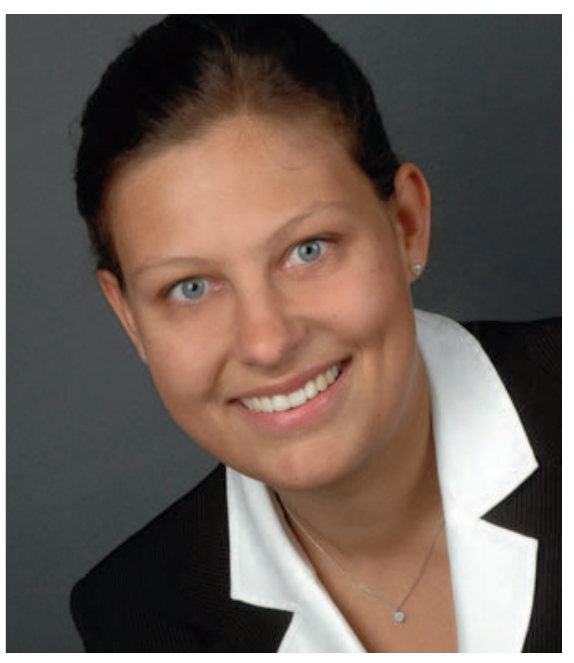

«Ein sehr praxisnahes und gut verständliches Tool»
NET und deren Behandlung an eine breite Ärzteschaft zu vermitteln. Das Programm verfügt über eine intuitive Bedienung und ein ansprechendes Design, inhaltlich wirkt der erste Fall gut durchdacht und realitätsnah. Die vorgegebene Reihenfolge der einzelnen Schritte entspricht nicht immer der eigenen Handhabung, z.B. würde ich die Bedeutung der Tumormarker im Behandlungsverlauf bzw. in der Nachsorge höher einschätzen als bei der Diagnosestellung, jedoch führt gerade dies zum Hinterfragen und Überdenken der eigenen Strategie, was ja ausdrücklich erwünscht ist. Interessant bei diesem speziellen Patientenfall wäre sicherlich der weitere Krankheitsverlauf mit der Frage nach Staging-Intervallen und weiteren Therapielinien. Auch könnte man über die Integration einer interdiszirapieentscheidung geführt. Man hat stets die Möglichkeit zwischen verschiedenen Bereichen und Untersuchungen auszuwählen, angefangen mit der körperlichen Untersuchung, Labordiagnostik, über bildgebende Verfahren wie Abdomensonographie oder CT-Diagnostik, bis hin zu endoskopischen und histopathologischen Untersuchungen. Die Befunde werden - jederzeit einsehplinären Tumorkonferenz nachdenken. Insgesamt hat die Bearbeitung des Programms Spaß gemacht und ich freue mich auf die weiteren Patientenfälle.

Bettina Otte, Berlin

Kontaktadresse: Bettina Otte, Charité - Universitätsmedizin Berlin, Augustenburger Platz 1, 13353 Berlin, Deutschland, bettina-susanne.otte@charite.de. 